\title{
A educação em ambientes virtuais: proposição de recursos computacio- nais para aumentar a eficiência do processo ensino-aprendizado
}

\author{
Lissandra Luvizão Lazzarotto \\ Instituto Federal de Educação, Ciência e Tecnologia do \\ Rio Grande do Sul (IFRS) \\ lissandra.lazzarotto@bento.ifrs.edu.br \\ José Luis Braga \\ Departamento de Informática da Universidade Federal de \\ Viçosa (UFV) \\ zeluis@dpi.ufv.br
}

\author{
Alcione de Paiva Oliveira \\ Departamento de Informática da Universidade Federal de \\ Viçosa (UFV) \\ alcione@dpi.ufv.br

\section{Frederico José Vieira Passos} \\ Coordenadoria de Educação Aberta e a Distância da Uni- \\ versidade Federal de Viçosa (UFV) \\ fvpassos@ufv.br
}

Resumo A informática pode ser considerada uma grande aliada no processo educacional, especialmente em ambientes virtuais de ensino e aprendizagem (AVEAs). Assim, tendo como foco básico a avaliação e os estilos de aprendizagem de alunos, o objetivo deste estudo é apresentar proposição baseada no emprego de recursos computacionais, que contribuam para aumentar a eficiência do processo ensino-aprendizado nesses ambientes. Para tanto, a partir de uma pesquisa de cunho aplicado, com características de naturezas bibliográfica e descritiva, foi desenvolvida proposição que contempla o cumprimento de cinco etapas fundamentais: 1) identificação dos estilos de aprendizagem dos alunos; 2) enquadramento dos estudantes em diferentes grupos, conforme os seus estilos de aprendizagem; 3) ajustes das formas de administração dos vários conteúdos programáticos, de acordo com os vários grupos formados de alunos; 4) efetuação de constantes avaliações da aprendizagem para medir a eficiência do processo ensinoaprendizado e adequar os estudantes aos diferentes grupos de estilos de aprendizagem; e 5) geração de relatórios e análises estatísticas, individuais e grupais, referentes a resultados de desempenho e estilos de aprendizagem dos alunos. Dentre as conclusões, destaca-se que a definição de estratégias de ensino a partir do conhecimento dos diferentes estilos de aprendizagem dos alunos pode constituir aspecto de grande relevância para melhorar o processo ensino-aprendizado em AVEAs.

Palavras-Chave: informática na educação, estilos de aprendizagem, modelagem computacional

\begin{abstract}
The computer science is a great allied in the education process, especially in virtual environments for teaching and learning (VETLs). Based on the evaluation of the learning and the students' learning styles, this study aims to present propositions that, by way operational procedures associated with the use of computational resources, contribute to increase the efficiency of the teaching-learning process in VETLs. We realized a applied research, with bibliographical and descriptive characteristics, to develop propositions that meets five basic steps: 1) identify the students' learning styles; 2) grouping students into different groups according to their learning styles; 3) adjusting the administration of educational content to the various groups of students; 4) make constant assessments of learning to measure the efficiency of the teaching-learning process and adapt the students to different groups of learning styles; and 5) produce reports and statistical analysis for individuals and groups about the results and performance associated to the students' learning styles. It is concluded that it is possible to define teaching strategies based in the students' learning styles to improve the teaching-learning process in the VETLs.
\end{abstract}

Keywords: computer science in the education, learning styles, computer modeling 


\section{Introdução}

A informática tem sido considerada, pela maioria dos profissionais da educação, psicologia e da ciência da computação, uma grande aliada no processo ensinoaprendizado. Entretanto, pesquisas têm demonstrado que o uso da informática na educação somente será eficaz com o desenvolvimento de ambientes educacionais, onde o computador seja utilizado como uma ferramenta de interação entre o aluno, o professor e o conteúdo a ser ensinado [16].

A grande expansão do uso da informática no processo educacional, ocorrida nos últimos anos, se deve principalmente aos custos mais acessíveis dos equipamentos e à disseminação de novas tecnologias da informação e das comunicações [1]. Dentre as tecnologias que mais se expandiram, têm-se as redes de computadores, com destaque especial para a Internet.

Com relação aos processos educacionais, pode-se ressaltar que eles estão intimamente ligados às formas de comunicação adotadas no ambiente instrucional, pois para se gerar conhecimento, em geral, é necessário promover a comunicação bidirecional entre o aprendiz e o tutor. Nessa linha, é possível inferir que as novas tecnologias de informação e comunicação e, em especial, os ambientes virtuais de ensino de aprendizagem (AVEAs), trazem novo vigor para as modalidades de ensino desenvolvidas de forma presencial e/ou a distância.

Um AVEA é um sistema computacional (ou software) desenvolvido para auxiliar a criação de cursos acessíveis, sobretudo, pela Internet. Esse tipo de software permite aos professores criar e conduzir cursos, mediante proposição de atividades pedagógicas (resolução de exercícios, provas, discussões de temas específicos, entre outros) e disponibilização de materiais didáticos organizados a partir de um plano de ensino. Os alunos, por sua vez, acessam o AVEA para responder as questões, discutir os temas propostos, consultar os materiais disponibilizados e interagir com os colegas e professores, buscando sanar suas dúvidas.

Os AVEAs podem ser utilizados em todos os níveis educacionais (fundamental, médio, graduação e pósgraduação), pois apresentam componentes semelhantes aos de uma sala de aula convencional. Entre esses componentes, destacam-se o professor, o aluno, os materiais didáticos e os processos de avaliação da aprendizagem e de interação.

Um ponto importante no estudo dos modelos de ensino, e um dos mais polêmicos, especialmente, em cursos de educação virtuais, relaciona-se à avaliação da aprendizagem. Isso porque um professor dificilmente consegue avaliar seus alunos de maneira adequada, a partir das características cognitivas dos mesmos. Esse problema é, talvez, um dos mais debatidos dentro do escopo de teorias e práticas, que compõem o estudo das ciências pedagógicas.

Para Massetto [23], é fundamental considerar a avaliação como um processo integrado ao processo de aprendizagem, pois, se bem planejada, pode funcionar como elemento motivador e incentivador da aprendizagem. Adicionalmente, ela pode constituir ferramenta essencial para avaliar a eficiência do processo ensino-aprendizado. Diante disso, é possível inferir que o processo de avaliação pode gerar conhecimentos importantes para o aluno e o professor.

Quando feita em ambientes virtuais, por exemplo, a avaliação tende a ser mais complexa, pois, em geral, não se tem o feedback, como ocorre nas interações face-aface, que possibilita uma avaliação subjetiva do aprendiz, dando indícios da compreensão e interesse deste [27]. Para minimizar esse problema, levar em conta os estilos de aprendizagem dos alunos [14] pode ser uma alternativa muito interessante. Conhecendo as diferentes maneiras como os alunos preferem aprender, o professor pode formar grupos mais homogêneos de estudantes e, assim, adequar as estratégias de ensino de acordo com os diferentes estilos de aprendizagem. Dentro dessa perspectiva, tendo como foco básico a avaliação e os estilos de aprendizagem de alunos, o objetivo deste estudo é apresentar proposição baseada no emprego de recursos computacionais, que contribuam para aumentar a eficiência do processo ensino-aprendizado em AVEAs.

Salienta-se que, mediante consultas junto à literatura especializada nos temas abordados neste trabalho, a realização do mesmo é plenamente justificável. Isso porque nota-se grande carência de estudos que associam avaliação e estilos de aprendizagem em AVEAs.

Quanto aos aspectos metodológicos, o presente trabalho caracteriza-se por uma pesquisa de cunho aplicado, na qual o investigador é motivado pela necessidade de contribuir, de forma prática, na busca de soluções para problemas concretos. Essa pesquisa, também, apresenta características de naturezas bibliográfica e descritiva, sendo esta de caráter exploratório [6]. Em termos operacionais, o estudo foi desenvolvido, principalmente, por meio de análises de trabalhos que fazem parte da literatura especializada nos temas abordados.

Para o desenvolvimento da proposição baseada no emprego de recursos computacionais, que possibilita atender uma disciplina ministrada em AVEA, foram considerados os principais aspectos que cercam um ambiente dessa natureza. Primeiramente, organizou-se a estrutura básica (disciplinas, conteúdos didáticos etc.) que, normalmente, determina o funcionamento dos cur- 
sos realizados nesse tipo de ambiente. A partir dessa estrutura, e tomando como referência os trabalhos realizados por Felder e Silverman [14], Felder e Soloman [15] e Felder [11], introduziram-se importantes requisitos computacionais funcionais. Esses requisitos relacionam-se, principalmente, com a avaliação da aprendizagem a partir da identificação dos estilos de aprendizagem dos alunos, de acordo com as várias dimensões teóricas desses estilos.

Cabe ressaltar que, embora seja uma proposta que não tenha sido implementada, espera-se que, na prática, tenha adequada aplicabilidade. Isso porque, de certa forma, seguiram-se os passos de estudos realizados por Cerqueira [5], Diniz [9], Granito [17], Oliveira et al. [25], Kalatzis [19] e Murad [24], que, ao implementaram proposições um tanto similares, obtiveram bons resultados.

\section{Trabalhos correlacionados}

Com base na literatura consultada, pôde-se constatar que, a maior parte dos trabalhos técnico-científicos que envolve avaliação e estilos de aprendizagem em AVEAs trata esses temas de forma isolada. Dentro dessa linha, a respeito dos estilos de aprendizagem, pode-se destacar quatro importantes estudos: Diniz [9], Oliveira et al. [25], Kalatzis [19] e Dias et al. [8]. Diniz [9] buscou verificar como os estilos de aprendizagem de estudantes matriculados em um curso de licenciatura em computação, ministrado a distância, influenciavam na forma como os alunos interagiam nos fóruns de discussão do ambiente virtual. Oliveira et al. [25] buscaram associar as competências profissionais, requeridas em determinada profissão, aos diferentes estilos de aprendizagem, como forma de proporcionar maiores chances de adaptação às situações do dia-a-dia e às exigências do mercado de trabalho. Kalatzis [19] realizou estudo para investigar a influência de alternativas instrucionais (inovações pedagógicas relacionadas com o método Problem-Based Learning) e dos estilos de aprendizagem no desempenho de estudantes de uma disciplina desenvolvida com o uso de plataforma de ensino a distância. Por fim, a partir de análises do ambiente de ensino-aprendizagem adaptativo na Web (AdaptWeb®), Dias et al. [8] propuseram um processo para identificar o estilo cognitivo de aprendizagem do aluno, que constitui o modo particular de perceber uma informação durante o processo de aprendizagem.

Quanto aos trabalhos envolvendo avaliação da aprendizagem em ambientes virtuais, inicialmente deve-se enfatizar que eles tendem a ser conduzidos dentro de duas linhas de pesquisas principais: i) desenvolvimento de questões e testes objetivos; e ii) acompanhamento de participações em atividades de aprendizagem online. $\mathrm{Na}$ primeira linha, merecem ser destacados os trabalhos desenvolvidos por Brusilovsky e Miller [4] e Brusilovsky et al. [3]. O estudo apresentado por Brusilovsky e Miller [4] propõe soluções baseadas no uso de banco de dados de questões. Esse banco deve armazenar informações importantes sobre as questões de maneira que o sistema de avaliação gere testes de acordo com os parâmetros solicitados pelo professor. Por sua vez, Brusilovsky et al. [3] propõem um framework de avaliação em camadas, em que as informações são coletadas a partir de análises da interação do aluno com o computador, sendo construído um modelo de usuário. Com base nesse modelo, é feita a adaptação das questões para a realização da avaliação da aprendizagem.

Sobre o acompanhamento de participações em atividades de aprendizagem online, destacam-se os estudos realizados por Emiliano [10] e Rocha et al. [27]. O trabalho elaborado por Emiliano [10] esteve voltado para o desenvolvimento de um modelo de ambiente para execução de avaliações remotas em educação a distância, baseado na tecnologia de sistemas multiagentes. Finalmente, Rocha et al. [27] apresentam as ferramentas InterMap e Acessos, desenvolvidas pelo grupo de pesquisadores do Projeto TelEduc, que apóiam a avaliação formativa.

\section{Fundamentação teórica}

Os pressupostos teóricos que nortearam a realização deste trabalho estão enquadrados em dois tópicos: a avaliação da aprendizagem e os estilos de aprendizagem.

\subsection{Avaliação da aprendizagem}

A avaliação da aprendizagem é uma atividade fundamental para qualquer organização educacional. A atividade de avaliar caracteriza-se como um meio subsidiário do crescimento. Isso porque cria a base para a tomada de decisão, que é o meio de encaminhar os atos subsequentes, na perspectiva da busca de melhores resultados [22].

A avaliação no seio da atividade de aprendizagem é uma necessidade, tanto para o professor quanto para o aluno. No que diz respeito ao professor, ela fornece os elementos de conhecimentos, que o permitem situar-se, de maneira mais correta e eficaz, em relação à ação de estímulos e de guia ao aluno. Pelo lado do aluno, permite verificar em que aspectos ele deve melhorar durante o 
seu processo de aprendizagem. De forma geral, a avaliação serve de informação para a melhoria não só do produto final, mas do processo de sua formação. Assim, se a avaliação falhar, não será possível dispor de orientação sobre a relação entre o plano e os resultados obtidos [7].

Em um ambiente de ensino presencial tradicional, os professores utilizam diversos meios formais e informais para determinar quanto e como os seus alunos estão aprendendo. Ao utilizar meios formais de avaliação, os professores aplicam provas, questionários, testes, exercícios, relatórios de laboratório e lições de casa. Essas técnicas ajudam o instrutor a estimar o progresso do aluno e atribuir notas relativas ao grau de conhecimento adquirido. Quanto à avaliação informal, ela é realizada pelo professor em sala de aula, mediante algumas técnicas: faz perguntas e incentiva à participação dos alunos; observa atentamente as perguntas e os comentários realizados por eles; e monitora as expressões faciais e corporais dos estudantes. Esse tipo de avaliação fornece feedbacks imediatos ao professor, possibilitando fazer ajustes no ensino [28].

A avaliação da aprendizagem pode, ainda, ser classificada, quanto à sua função, em diagnóstica, somativa ou formativa. É diagnóstica quando precede à ação de formação, ou seja, quando é aplicada no início da aprendizagem, antes de ser apresentado o conteúdo ao aluno. Essa avaliação tem a função de permitir um ajuste recíproco aprendiz/programa de estudos. Esse ajuste pode ocorrer por modificação do programa, que será adaptado aos aprendizes, ou por orientação dos aprendizes para subsistemas de formação mais adaptados a seus conhecimentos e competências atuais [18].

A avaliação que ocorre após a ação de formação é conhecida como somativa, que tem o propósito de classificar os alunos, ao final de um período de aprendizagem, de acordo com os níveis de aproveitamento [2]. Ela tem a função de certificar aquisições em relação a terceiros, fornecendo poucos detalhes dos saberes e das competências adquiridas e do nível de domínio precisamente atingido em cada campo abrangido [26].

Por sua vez, a avaliação formativa permite constatar se os alunos estão, de fato, atingindo os objetivos pretendidos. Para isso, analisa-se a compatibilidade entre tais objetivos e os resultados efetivamente alcançados durante o desenvolvimento das atividades propostas. Para Bloom et al. [2], esse tipo de avaliação visa a informar, ao professor e ao aluno, sobre o rendimento da aprendizagem no decorrer das atividades escolares e a localização das deficiências na organização do ensino para possibilitar correção e recuperação.

De acordo com Hadji [18], qualquer avaliação tem uma dimensão cumulativa, pois na maioria das vezes se faz o balanço das aquisições dos alunos. Adicionalmente, o autor infere que todas as avaliações deveriam ter, em um contexto pedagógico, uma dimensão prognóstica, no sentido de conduzir a um melhor ajuste no processo ensino-aprendizado. Esse ajuste possibilita melhor adaptação do conteúdo e das formas de ensino às características dos alunos, que foram reveladas pela avaliação.

\subsection{Estilos de aprendizagem}

Conceitualmente, o termo "estilo de aprendizagem" se refere a forma como o aprendiz prefere perceber, reter, processar e organizar o conhecimento [9] e [25].

Os alunos podem aprender de diversas maneiras: vendo e ouvindo; refletindo e agindo; raciocinando lógica e intuitivamente; memorizando e visualizando. Os métodos de ensino, também, variam, pois os instrutores utilizam de diversas formas para repassar o conhecimento: alguns lêem, enquanto outros discutem ou demonstram; alguns focalizam em princípios e outros em aplicações [14]. Assim, professores e alunos tendem a realizar suas funções de ensinar e aprender segundo seus estilos de ensino e aprendizagem, que podem divergir e/ou convergir em sala de aula.

Estudantes cujos estilos de aprendizagem são compatíveis com o estilo de ensino do professor tendem a reter as informações por mais tempo e aplicar os conhecimentos adquiridos de forma mais efetiva [12]. Por outro lado, quando os alunos não conseguem aprender o que está sendo ensinado, podem ficar entediados e distraídos, e, como consequência, tiram notas baixas em provas, ficam desanimados com o curso, com o currículo e/ou com eles mesmos e pensam em mudar de curso ou abandonar os estudos. Os professores, diante de notas baixas e alunos que não participam ou apresentam baixa frequência, percebem que algo está errado, podendo ficar defensivos ou hostis e até questionar se estão na profissão certa $[13,14]$.

Esses problemas na relação professor/aluno podem, de certa forma, ser minimizados se os professores modificarem os seus estilos de ensino, de maneira a acomodar os estilos de aprendizagem de seus estudantes [13].

Felder [11] e Felder e Silverman [14] sintetizaram as conclusões de várias pesquisas para formularem um modelo de estilos de aprendizagem. Um modelo dessa natureza classifica os estudantes a partir da maneira como eles se adaptam, recebem e processam as informações. As dimensões dos estilos de aprendizagem e de ensino, propostas por esses autores, estão apresentadas no Quadro 1. 
As dimensões de estilos de aprendizagem do modelo em questão (sensitiva/intuitiva ${ }^{1}$, visual/verbal, ati$\mathrm{va} / \mathrm{reflexiva}^{2}$ e sequencial/global), aparentemente dicótomas, são contínuas. A preferência de um estudante, numa dada escala (por exemplo, por percepção sensitiva ou intuitiva), pode ser forte, moderada ou quase inexistente. Além disso, essa preferência pode mudar com o tempo, variando de acordo com o assunto ou o ambiente de ensino $[12,13]$.

As dimensões mostradas no Quadro 1 podem definir 16 tipos distintos de estudantes, de acordo com seus estilos de aprendizagem, que podem ser identificados a partir de respostas a quatro questões:

1. preferencialmente, que tipo de informação o estudante percebe: sensoriais (visões, sons, sensações físicas) ou intuitivas (memórias, idéias, introspecção)?

2. por meio de que modalidade a informação sensorial é mais eficientemente percebida: visual (figuras, diagramas, gráficos, demonstrações) ou verbal (sons, palavras faladas e escritas e fórmulas)?

3. como o estudante prefere processar a informação recebida: ativamente (mediante atividades físicas ou discussões) ou refletivamente (por meio de introspecção)? e

4. como o estudante progride na direção da compreensão: sequencialmente (em uma progressão lógica de pequenos passos incrementais) ou globalmente (em grandes saltos, holisticamente)? [14].

\begin{tabular}{|c|c|}
\hline $\begin{array}{c}\text { Estilos de aprendizagem } \\
\text { preferida }\end{array}$ & $\begin{array}{l}\text { Estilos de ensino } \\
\text { correspondente }\end{array}$ \\
\hline $\left.\begin{array}{l}\text { Sensitivo } \\
\text { Intuitivo }\end{array}\right\}$ Percepção & $\left.\begin{array}{l}\text { Concreto } \\
\text { Abstrato }\end{array}\right\}$ Conteúdo \\
\hline $\left.\begin{array}{l}\text { Visual } \\
\text { Verbal }\end{array}\right\} \mathrm{I}$ & $\left.\begin{array}{l}\text { Visual } \\
\text { Verbal }\end{array}\right\}$ Apresentação \\
\hline $\left.\begin{array}{l}\text { Ativo } \\
\text { Reflexivo }\end{array}\right\}$ Processamento & $\left.\begin{array}{l}\text { Ativo } \\
\text { Passivo }\end{array}\right\}$ Participação \\
\hline $\left.\begin{array}{l}\text { Sequencial } \\
\text { Global }\end{array}\right\}$ Compreensão & $\left.\begin{array}{l}\text { Sequencial } \\
\text { Global }\end{array}\right\}$ Perspectiva \\
\hline
\end{tabular}

Quadro 1: Dimensões de estilos de aprendizagem e de ensino. Fonte: Elaborado a partir de Felder e Silverman [14] e Felder [11]

\footnotetext{
${ }^{1}$ Sensitiva/intuitiva são categorias derivadas da teoria de tipos psicológicos de Carl Jung. O grau de preferência por sensação ou intuição pode ser determinado pelo indicador de tipos de Myers-Briggs [20].

2 Ativa/reflexiva é um componente de um modelo de aprendizagem desenvolvido por Kolb (1984), citado por Felder e Silverman [14].
}

Para os estilos de aprendizagem e de ensino, o modelo original, proposto por Felder e Silverman [14], possuía, também, a dimensão indutiva/dedutiva, que foi extinta por Felder [11]. Essa extinção deve-se ao fato de que o autor define a indução como o método mais adequado de ensino, principalmente em nível de graduação. Assim, considera que é melhor omitir essa dimensão do modelo para evitar que sirva como justificativa para os professores continuarem a usar, em suas aulas, o modo dedutivo, que é o preferido pelos alunos.

Operacionalmente, para determinar os estilos de aprendizagem dos estudantes, Felder e Soloman [15] desenvolveram o ILS (Index of Learning Styles), cujo objetivo era investigar e identificar as preferências de aprendizagem em diferentes dimensões. O ILS é composto por 44 itens, sendo 11 para cada uma das quatro dimensões supracitadas.

\section{A proposta para AVEAs}

Para discutir e apresentar os aspectos fundamentais da proposta que, por meio do emprego de recursos computacionais, busca contribuir para o aumento da eficiência do processo ensino-aprendizado em AVEAs, foram elaboradas as seções 4.1 e 4.2. Enquanto a primeira trata dos aspectos gerais, a seção 4.2 enfoca os aspectos específicos da proposta.

Em termos lógicos, a partir do emprego de recursos computacionais, a proposição contempla o cumprimento de algumas etapas fundamentais no processo ensinoaprendizado. Sumariamente, pode-se destacar que essas etapas procuram atender cinco grandes objetivos: 1) identificar os estilos de aprendizagem dos alunos; 2) enquadrar os estudantes em diferentes grupos, conforme os seus estilos de aprendizagem; 3) ajustar as formas de administração dos vários conteúdos programáticos, conforme os vários grupos formados de alunos; 4) efetuar constantes avaliações da aprendizagem para medir a eficiência do processo ensino-aprendizado e adequar os estudantes aos diferentes grupos de estilos de aprendizagem; e 5) gerar relatórios e análises estatísticas, individuais e grupais, referentes a resultados de desempenho e estilos de aprendizagem dos alunos.

\subsection{Aspectos gerais da proposta}

A maioria dos cursos ministrados em AVEAs é constituída por uma ou mais disciplinas. Cada disciplina é organizada por um ou mais conteúdos didáticos. $\mathrm{O}$ conteúdo didático envolve a elaboração e a aplicação de várias atividades, como: apresentação de conceitos, rea- 
lização de avaliações da aprendizagem por meio de exercícios, testes e provas, discussões de temas em salas de bate-papos (chats) e postagens de trabalhos em portfólio.

A atividade "Avaliação", associada com a proposição de exercícios, testes e provas, pode ser realizada com questões objetivas e/ou subjetivas. Porém, para AVEAs, a preferência tem sido pelas avaliações objetivas, projetadas para uma única resposta correta, possibilitando a automatização das correções e a rápida entrega dos resultados.

É relevante destacar que a proposição de exercícios, testes e provas não é a única forma de avaliar a aprendizagem dos alunos nos ambientes em questão. Isso porque a maioria desses ambientes dispõe de importantes ferramentas (fórum de discussão, sala de bate-papo, portfólio, correio eletrônico etc.), que permitem realizar avaliações contínuas da aprendizagem dos alunos. A avaliação da aprendizagem por meio dessas outras ferramentas pode ser feita de forma quantitativa e qualitativa.

Cabe assinalar, ainda, que, para atender os objetivos institucionais e diminuir a complexidade no desenvolvimento da proposta em discussão, visando a torná-la viável para uma futura implementação, as avaliações da aprendizagem estarão associadas com a proposição de questões objetivas.

Apesar de limitações práticas, se considera que, para contribuir com a melhoria do processo ensinoaprendizado, as referidas avaliações podem ser utilizadas como referências para identificar e atualizar os estilos de aprendizagem dos alunos.

Por meio do processo de avaliação, é possível identificar os diferentes estilos de aprendizagem, que seguem a classificação estabelecida no modelo desenvolvido por Felder e Silverman [14]. A partir de estudos realizados por esses autores, elaborou-se a proposta para AVEAs, que contempla várias etapas (Figura 1). Foi estabelecido que a identificação inicial dos estilos de aprendizagem deve ocorrer na etapa definida como Avaliação de Identificação dos Estilos de Aprendizagem (AIEA). Nessa etapa se aplica o Index of Learning Styles (ILS), que pode resultar em 16 diferentes grupos de alunos, conforme demonstrado no Quadro 2.

Ao analisar os agrupamentos potenciais formados a partir da aplicação do ILS, pode-se inferir que é praticamente inviável, para o professor, elaborar conteúdos, avaliações (provas, testes e exercícios), chats e fóruns de discussão para 16 distintos grupos potenciais de alunos. Para Felder [14], a maioria dos professores pode se sentir intimidada ao tentar acomodar, simultaneamente e numa única classe, todos esses grupos diferentes de alunos.
Diante dessas dificuldades, visando a viabilizar o uso dos estilos de aprendizagem de forma que possa contribuir, efetivamente, para melhorar o trabalho do professor, na proposta apresentada neste estudo, optou-se por levar em conta apenas duas dimensões associadas com esses estilos: intuitiva/sensitiva e global/sequencial. Como propõe-se identificar, para AVEAs, os diferentes estilos dos alunos por meio da realização de avaliações convencionais individuais (provas, testes e exercícios), justifica-se utilizar apenas essas duas dimensões. Isso porque, de acordo com a literatura especializada, os resultados de uma avaliação convencional podem ser muito influenciados, sobretudo, pelas características inerentes aos estilos intuitivo, sensitivo, global e sequencial.

Portanto, nas discussões específicas sobre a proposição, realizadas na seção 4.2, por duas razões principais, dá-se pouca ênfase às dimensões ativa/reflexiva e verbal/visual. A primeira razão está relacionada com o fato de que, durante a realização de uma avaliação convencional, em geral, o estilo ativo praticamente não se manifesta, pois, normalmente, não ocorrem discussões e nem realização de trabalhos em grupo, que são características essenciais desse estilo. Ao desconsiderar o estilo ativo, justifica-se, também, não levar em conta o estilo reflexivo, pois, juntamente com o primeiro, forma a dimensão ativa/reflexiva. A segunda razão está associada com o estilo verbal, cuja manifestação tende a ser pequena em avaliações convencionais. Isso porque os aprendizes verbais têm preferência pela fala e audição, que normalmente não podem ser utilizadas nesses tipos de avaliações. Como consequência da exclusão do estilo verbal, é necessário desconsiderar também o seu estilo oposto (visual). Assim, ao levar em conta somente as dimensões intuitiva/sensitiva e global/sequencial, o professor, ao invés de preparar atividades de avaliação para 16 grupos potenciais de alunos, terá o trabalho de elaborar, no máximo, quatro formas de avaliações distintas.

Embora a proposta foi estruturada para considerar, basicamente, apenas as duas dimensões referidas, é importante ressaltar que, inicialmente, no processo de identificação e seleção das diferentes dimensões de estilos de aprendizagem, são levadas em conta as quatro dimensões. Esse processo, ilustrado na Figura 1, tem início com a Avaliação de Identificação dos Estilos de Aprendizagem (AIEA), em que os alunos são submetidos ao ILS. Com base nas respostas fornecidas pelos estudantes, são calculados os seus estilos predominantes para, então, enquadrá-los entre os 16 grupos potenciais, que formarão a Base de Conhecimento Inicial dos Estilos de Aprendizagem (BCIEA).

A BCIEA é a referência inicial, que possibilita, ao professor, planejar e elaborar as atividades pedagógicas 
iniciais de acordo com os estilos de aprendizagem dos alunos. Porém, conforme já salientado, para tornar viável a utilização desses estilos por parte do professor, na proposição elaborada neste trabalho, após a BCIEA, é realizada a Seleção dos Estilos de Aprendizagem (SEA). A partir dessa etapa, passam a ser contempladas apenas as dimensões Intuitiva/Sensitiva e Global/Sequencial.

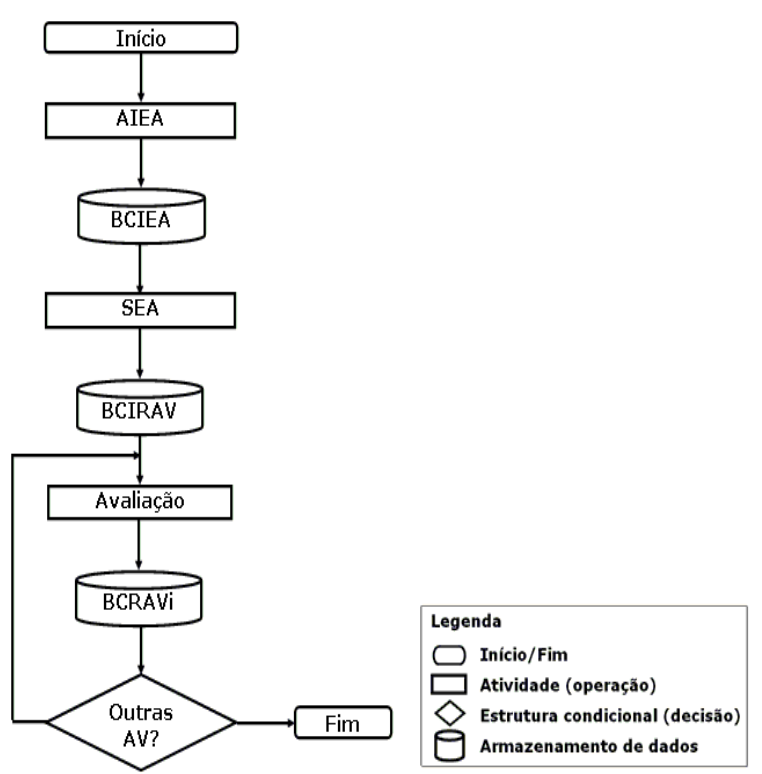

Figura 1: Processo de identificação e seleção das diferentes dimensões de estilos de aprendizagem por meio de avaliações.

Fonte: Elaborado a partir de Felder e Silverman [14] e Felder [11].

Após a SEA, forma-se uma nova base de conhecimento dos estilos de aprendizagem, que pode ser utilizada para o desenvolvimento efetivo das diferentes atividades pedagógicas. Considerando a atividade Avaliação, o professor poderá utilizar, como referência, a Base de Conhecimento Inicial e Referencial para Avaliação da Aprendizagem (BCIRAV), que é formada por quatro grupos distintos de alunos.

Conforme enfatizado por Felder [12], a preferência de um aluno por um determinado estilo de aprendizagem pode mudar com o tempo ou variar de acordo com o assunto ou ambiente de ensino. Assim, é importante que essa preferência seja constantemente reavaliada no decorrer das atividades desenvolvidas em uma disciplina.

O resultado alcançado na avaliação da aprendizagem pode ser considerado um bom indicador para verificar se o aluno está, ou não, aprendendo satisfatoriamente dentro dos seus estilos preferidos. Nessa perspectiva, propõe-se adequações no ensino em que, mediante os resultados obtidos com a resolução de exercícios, testes e/ou provas, sejam efetuadas constantes reavaliações para confirmar ou reagrupar os alunos, de acordo com seus estilos de aprendizagem. Assim, cada vez que o aluno resolver um exercício, teste ou prova, seus estilos de aprendizagem identificados são reavaliados, gerando uma nova Base de Conhecimento Referencial de Avaliação $\left(\mathrm{BCRAV}_{\mathrm{i}}\right.$, onde $i$ indica a versão da base) (Figura $1)$.

Levando em conta que os estilos de aprendizagem identificados nas diversas avaliações tendem a ser preferidos, também, para realizar outras atividades (por exemplo, conteúdo didático, fórum de discussão e chats), cada alteração realizada na $\mathrm{BCRAV}_{\mathrm{i}}$ pode ser utilizada para efetuar ajustes nas formas de desenvolver essas demais atividades. Nesse sentido, especialmente em relação ao conteúdo didático, pelo fato de anteceder à avaliação da aprendizagem, é importante que a forma de administrá-lo esteja sempre ajustada aos diferentes estilos de aprendizagem dos alunos.

\subsection{Aspectos específicos da proposta}

Para tratar de aspectos mais específicos da proposição, foram construídas duas subseções. Na primeira, são efetuadas discussões envolvendo questões estruturais. $\mathrm{Na}$ segunda, apresentam-se alguns indicadores estatísticos, que podem ser obtidos a partir dos históricos derivados da implementação da proposta.

\subsubsection{Questões estruturais}

A estrutura da proposta a ser utilizada para uma disciplina ministrada em AVEA, de acordo com a Figura 2, está organizada em várias etapas. $\mathrm{Na}$ etapa inicial (Etapa 1) realiza-se a Avaliação de Identificação dos Estilos de Aprendizagem (AIEA) dos alunos, que ocorre antes de administrar os conteúdos programáticos da disciplina. Os resultados da AIEA, com 16 grupos potenciais de alunos, formam a Base de Conhecimento Inicial dos Estilos de Aprendizagem (BCIEA).

A partir da BCIEA, efetua-se a Seleção dos Estilos de Aprendizagem (SEA), que passa a contemplar quatro grupos distintos de alunos: grupo 1, formado por estudantes classificados como intuitivos (INT) e globais (GLO); grupo 2, constituído pelos alunos definidos como intuitivos (INT) e sequenciais (SEQ); grupo 3, composto pelos estudantes considerados sensitivos (SEN) e globais (GLO); e grupo 4, representado pelos estudantes identificados como sensitivos (SEN) e sequenciais (SEQ). Esses quatro grupos formam a Base de Conhecimento Inicial e Referencial para Avaliação da Aprendi- 
zagem (BCIRAV), que representa a referência inicial para executar a Etapa 2.

Nas etapas seguintes do modelo, a representação dos quatro grupos é feita por meio da sigla AVEn, seguida do número do grupo. Por exemplo, na Etapa 2, o grupo 1 é representado pela sigla $\mathrm{AV} 2 \mathrm{n}(1)$, em que AV indica avaliação da aprendizagem, 2 é o número da Etapa de onde é originada a base para a avaliação corrente, $n$ é o número da avaliação dentro da etapa atual e (1) refere-se ao número de identificação do grupo. Para os demais grupos, segue-se a mesma forma de representação.

O modelo em discussão prevê, ainda, a coleta e o armazenamento de dados individuais e grupais dos alunos. Para isso, são coletadas e armazenadas várias informações, como: nome, idade, matrícula e notas de cada aluno; número total de alunos presentes em determinado grupo; percentual de alunos presentes no grupo em relação ao total de alunos matriculados na disciplina; e notas médias dos grupos de alunos. Com os dados coletados durante as avaliações da aprendizagem, que são realizadas no decorrer do desenvolvimento da disciplina, é possível efetuar análises estatísticas, que visam a auxiliar o professor na elaboração das próximas atividades pedagógicas.

Os exercícios, testes e provas devem ser elaborados de acordo com o conteúdo programático $(\mathrm{CP})$, que foi repassado aos alunos durante determinado período. Uma disciplina pode ser dividida em vários conteúdos programáticos. Essa divisão depende, geralmente, da extensão dos assuntos tratados e da forma como o professor prefere trabalhar esses assuntos. Desse modo, o número total de etapas do modelo proposto é igual ao número de conteúdos programáticos mais $1(\mathrm{CPs}+1)$.

Na Etapa 2, realiza-se a administração do primeiro conteúdo programático (CP1). Tanto o $\mathrm{CP}$ como a primeira avaliação são elaborados usando como referência a BCIRAV, que foi definida na Etapa 1.

Considerando que o mesmo CP pode ser avaliado mais de uma vez, um número (n) é gerado para cada avaliação da aprendizagem (AV). Assim, na Etapa atual a representação da avaliação corrente é feita por AVn.

Em relação às questões para a avaliação dos $\mathrm{CPs}$, considera-se que o professor pode elaborá-las para todos os grupos de alunos. No entanto, deve formulá-las de acordo com os estilos de aprendizagem de cada grupo. Para auxiliar na compreensão dessa formulação, a seguir apresenta-se, como exemplo, o enunciado de uma questão referente ao conceito de "Sistema", que pode ser elaborado de quatro formas diferentes, conforme os distintos estilos de aprendizagem:

1) Estilos Sensitivo/Sequencial: Sistema é um conjunto de elementos dinamicamente relacionados entre si, que formam um todo unitário ou complexo, com a finalidade de atingir um propósito (ou objetivo), mediante operações de entradas (informação, energia ou matéria) e fornecimento de saídas (informação, energia ou matéria) que foram processadas. Assim, um sistema é formado por cinco parâmetros principais. Assinale a alternativa que apresenta, de forma ordenada, os cinco parâmetros de um sistema.

2) Estilos Sensitivo/Global: Sistema é um conjunto de elementos dinamicamente relacionados entre si, que formam um todo unitário ou complexo, com a finalidade de atingir um propósito (ou objetivo), mediante operações de entradas (informação, energia ou matéria) e fornecimento de saídas (informação, energia ou matéria) que foram processadas. Assim, um sistema é formado por cinco parâmetros principais. Lembre-se de que os parâmetros apresentados pelo referido autor são aplicados, também, em sistemas biológicos (corpo humano, por exemplo), sistemas educacionais, sistemas computacionais, sistema solar. Com base nisso, assinale a alternativa que apresenta os cinco parâmetros que são encontrados em qualquer sistema.

3) Estilos Intuitivo/Sequencial: Leia as alternativas abaixo e assinale aquela que apresenta, de forma ordenada, os cinco parâmetros que compõem qualquer sistema.

4) Estilos Intuitivo/Global: Leia as alternativas abaixo e assinale aquela que se refere aos cinco parâmetros que compõem um sistema de forma ordenada. Considere que os referidos parâmetros compõem qualquer tipo de sistema, como, por exemplo, o biológico, solar, educacional e empresarial.

Além da formulação de questões que respeitem os distintos estilos de aprendizagem, é importante determinar e informar, aos alunos, o tempo para responder cada questão. Isso porque o tempo constitui, também, um dos fatores relevantes associados com as preferências dos estilos de aprendizagem dos estudantes. 

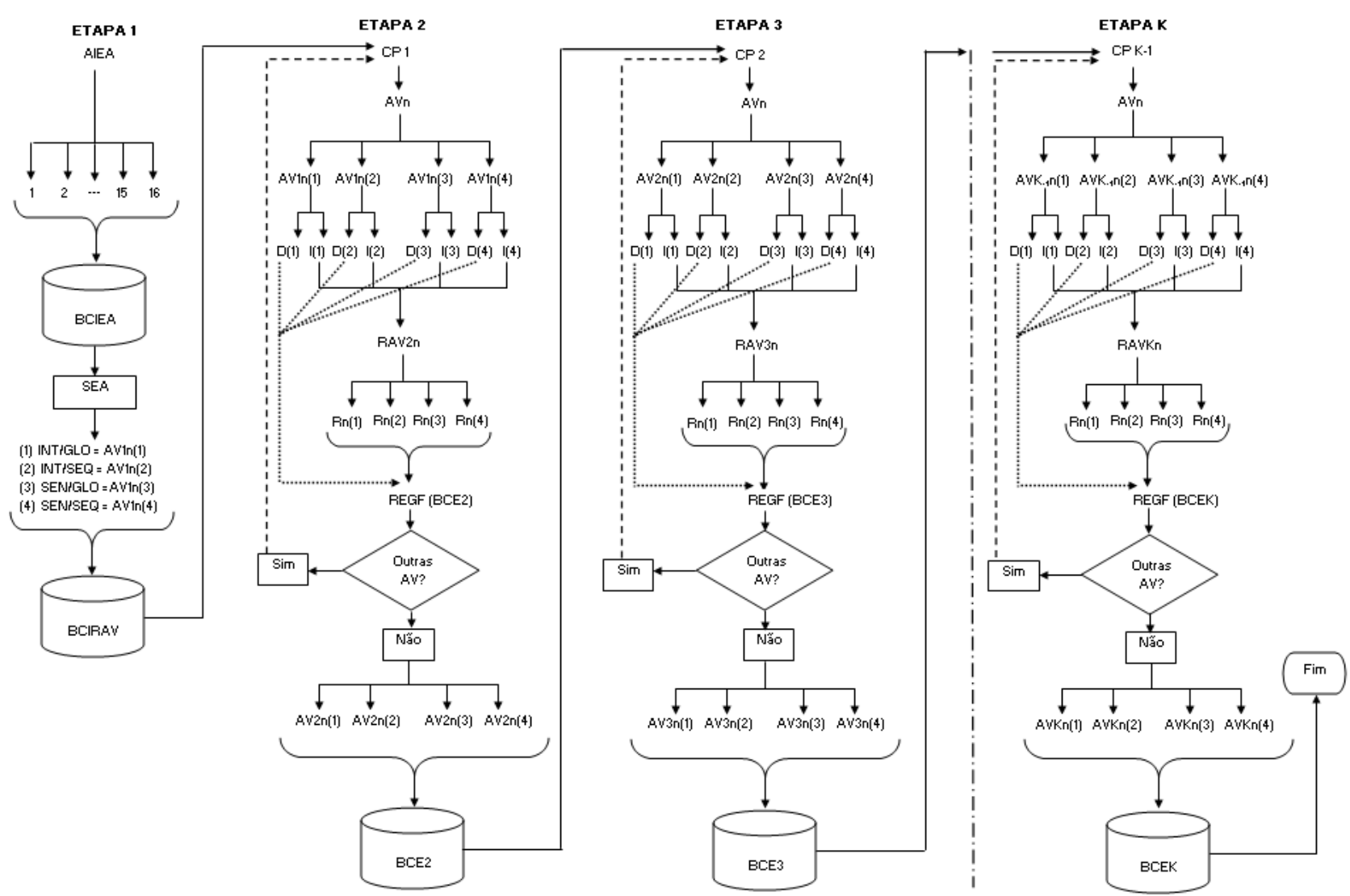

Figura 2: Síntese da proposição para uma disciplina ministrada em AVEA.

Após cada avaliação ser finalizada e corrigida, os alunos de cada grupo são classificados em duas categorias: definidos (D) e indefinidos (I). Os alunos que pertencem à categoria definidos são aqueles que, na avaliação, obtiveram nota igual ou superior à média determinada pela instituição de ensino. Com esse desempenho, se assume que esses estudantes estão definidos quanto aos seus estilos de aprendizagem, devendo, portanto, permanecer nos mesmos grupos que foram previamente enquadrados. Por outro lado, os alunos pertencentes à categoria indefinidos são aqueles que não atingiram a média na avaliação. Nesse caso, é necessário verificar se o baixo desempenho na avaliação pode estar, ou não, vinculado a não adaptação dos alunos aos estilos de aprendizagem em que estavam agrupados.

Para diagnosticar os problemas que os alunos da categoria indefinidos encontraram durante a avaliação, realiza-se uma auto-avaliação, onde eles devem analisar cada questão errada e assinalar quais foram as suas dificuldades. A auto-avaliação é denominada, no modelo proposto, por Reavaliação da Avaliação, que é representada pela sigla RAV, seguida do número da etapa e do número (n) da avaliação. Na Etapa 2, por exemplo, ela aparece como RAV2n.

Como a RAV é aplicada para os alunos considerados indefinidos em cada grupo, ela passa a ter, também, uma representação para cada grupo. Essa representação é dada pela letra $\mathrm{R}$, seguida do número (n) da avaliação e do número do grupo. A reavaliação do grupo 1, por exemplo, é representada por $\mathrm{Rn}(1)$.

$\mathrm{Na}$ reavaliação, inicialmente, apresenta-se para o aluno a primeira questão errada. Após uma breve análise, o aluno responde à seguinte pergunta: "Conhecia o assunto tratado na questão?". Se a resposta do aluno for "Não", considera-se que ele não estudou o assunto e, nesse caso, não tem como avaliar outras dificuldades. Com isso a análise da questão é finalizada. Por outro lado, se o aluno responder "Sim", entende-se que ele encontrou outras dificuldades para resolver a questão. Assim, faz-se necessário investigar se os seus estilos de aprendizagem foram corretamente identificados, pois podem estar afetando negativamente o seu desempenho. Nesse caso, deve-se dar continuidade à RAV, visando a levantar os problemas encontrados pelo aluno durante a realização da avaliação. 
Para continuar a RAV, são apresentadas algumas alternativas para o aluno (Quadro 2). Essas alternativas foram elaboradas com base nos estudos realizados por Felder e Silverman [14]. Para cada questão errada, o aluno deve escolher as alternativas que mais se aproximam das dificuldades encontradas na resolução da questão. Destaca-se que, cada alternativa apresentada ao aluno está relacionada a um estilo de aprendizagem das duas dimensões (sensitiva/intuitiva ou glo$\mathrm{bal} /$ sequencial). Dessa forma, o estudante poderá escolher apenas uma alternativa referente a determinada dimensão. Se ele escolher, por exemplo, a alternativa 1 para a dimensão Sensitiva/Intuitiva e a alternativa 2 para a dimensão Global/Sequencial, suas escolhas, para determinada questão, estariam associadas aos estilos, respectivamente, sensitivo e sequencial. As alternativas escolhidas são armazenadas para que sejam efetuadas análises no final da RAV.

\begin{tabular}{|c|l|c|}
\hline \multicolumn{4}{|c|}{ DIMENSÃO SENSITIVA/INTUITIVA } \\
\hline $\begin{array}{c}\text { Número } \\
\text { da } \\
\text { alternativa }\end{array}$ & Descrição da alternativa para RAV & $\begin{array}{c}\text { Estilo de } \\
\text { aprendizagem } \\
\text { correspondente }\end{array}$ \\
\hline 1 & $\begin{array}{l}\text { Faltou tempo para resolver a } \\
\text { questão ou não a entendeu. Ela } \\
\text { deveria ser mais detalhada. }\end{array}$ & Sensitivo \\
\hline 2 & $\begin{array}{l}\text { Não teve paciência para ler a } \\
\text { questão, ela é muito extensa e } \\
\text { muito detalhada ou não releu a } \\
\text { questão para ver se estava } \\
\text { realmente correta. }\end{array}$ & Intuitivo \\
\hline $\begin{array}{c}\text { DIMENSÃO GLOBALISEQUENCIAL } \\
\text { da }\end{array}$ & $\begin{array}{l}\text { Descrição da alternativa para RAV } \\
\text { alternativa }\end{array}$ & $\begin{array}{l}\text { Eprendizagem } \\
\text { correspondente }\end{array}$ \\
\hline 1 & $\begin{array}{l}\text { Teve dificuldades para entender a } \\
\text { questão. Essa questão é muito } \\
\text { limitada, necessita compreender o } \\
\text { todo, ver as outras conexões com o } \\
\text { assunto. }\end{array}$ & \multicolumn{1}{|c|}{ Global } \\
\hline 2 & $\begin{array}{l}\text { A questão mostra um quadro geral, } \\
\text { muitos detalhes foram ocultados, } \\
\text { necessita que o assunto seja } \\
\text { mostrado passo a passo. }\end{array}$ & Sequencial \\
\hline
\end{tabular}

Quadro 2: Alternativas para a realização da RAV, que estão associadas com as questões erradas.

Fonte: Elaborado a partir de Felder e Silverman [14].

Após finalizar a reavaliação de todas as questões erradas, os dados coletados são processados por recursos computacionais, gerando automaticamente indicadores estatísticos. Primeiramente, é verificado o percentual das questões erradas em que o aluno assinalou que não conhecia o assunto tratado. Um valor superior a $50 \%$ é forte indicativo de que o mau desempenho na avaliação deve-se à falta de dedicação aos estudos. Nesse caso, o aluno deve permanecer no mesmo grupo até que seja submetido a uma nova avaliação do $\mathrm{CP}$. Por outro lado, se o referido valor for igual ou inferior a $50 \%$, é possível presumir que, as dificuldades do aluno podem estar relacionadas com os estilos de aprendizagem em que estava enquadrado nas atividades realizadas. Nessa situação, é necessário analisar os resultados obtidos nas alternativas assinaladas pelos alunos, durante a realização da RAV.

Os resultados da RAV fornecem informações referentes às dificuldades encontradas pelo aluno durante a avaliação. Essas dificuldades podem estar vinculadas a duas situações principais: 1) a sua preferência pelos estilos de aprendizagem sofreu alteração e, por isso, ele não está conseguindo se adaptar aos estilos em que foi classificado; ou 2) a sua preferência pelos estilos não sofreu alteração e o problema pode estar na avaliação, que não foi elaborada de acordo com os seus estilos de aprendizagem.

Para identificar o problema que pode estar contribuindo para o mau desempenho do aluno, inicialmente analisa-se o resultado percentual de cada estilo de aprendizagem obtido na reavaliação. Se houver igualdade nos resultados percentuais (por exemplo: $50 \%$ sensitivo e $50 \%$ intuitivo; e $50 \%$ global e $50 \%$ sequencial), o aluno deve permanecer no mesmo grupo que vinha trabalhando. Por outro lado, não havendo essa igualdade, efetua-se uma verificação de quais estilos de aprendizagem tiveram maior percentagem de ocorrência. Se os estilos forem diferentes daqueles que o aluno vinha trabalhando, considera-se que a sua preferência pelos mesmos sofreu alteração, e, portanto, ele deve ser reagrupado. Caso contrário, o aluno mantém sua preferência, permanecendo, assim, no mesmo grupo. É importante assinalar que, mesmo na situação em que não ocorrer mudanças na preferência, o professor deve avaliar as alternativas marcadas pelo aluno e buscar minimizar as dificuldades encontradas por ele.

Após identificar as dificuldades dos alunos, definindo a permanência, ou não, em determinados grupos, o modelo proposto realiza um Reagrupamento Final (REGF). O REFG corresponde à reorganização, em novos grupos, dos alunos definidos e reavaliados, formando, com isso, a nova Base de Conhecimento Referencial de Avaliação na Etapa, que é representada pela sigla BCE, seguida do número da etapa. Essa base servirá como referência para a próxima avaliação (AV), que pode vir a ser realizada dentro da etapa atual, ou para iniciar a etapa subsequente.

Da Etapa 2 até a Etapa K (etapa de administração do último $\mathrm{CP}$ ), os passos e procedimentos propostos são os mesmos. A finalização do processo ocorre quando se realiza a última avaliação associada com o último CP da Etapa K (Figura 2). 


\subsubsection{Geração de indicadores estatísticos}

Os dados gerados em cada etapa são armazenados de forma a gerar um histórico, que contém informações relacionadas aos estilos de aprendizagem dos alunos, bem como da distribuição destes nos diferentes grupos. Portanto, após finalizar todas as etapas, pode-se obter um histórico geral do conjunto total de informações geradas. A partir desse conjunto, podem ser produzidos, de forma automática, relatórios com importantes indicadores estatísticos individuais e grupais (Figura 3). Esses relatórios podem ser apresentados em forma de gráficos e tabelas.

Com relação aos indicadores estatísticos individuais, podem ser obtidos a média, o desvio padrão e o coeficiente de variação das notas alcançadas nas avaliações da aprendizagem. Além da média das notas, é importante levar em conta medidas relacionadas com a dispersão dos valores. Essas medidas são representadas pelo desvio padrão (DP) e pelo coeficiente de variação (CV).

Com o DP e o CV, é possível analisar a variabilidade das notas do aluno ao longo do desenvolvimento das várias etapas da disciplina. Adicionalmente, com base no $\mathrm{CV}$, o professor pode, de acordo com o grau de variabilidade no desempenho, classificar os alunos em diferentes grupos. Essa classificação visa a desenvolver estudos mais específicos acerca das razões que levam determinados grupos de estudantes a apresentar desempenhos mais ou menos variáveis.

Quanto aos estilos de aprendizagem individuais, que foram detectados ao longo de toda a disciplina, pode-se, também, efetuar análises do comportamento das preferências. Nessa perspectiva, é possível avaliar, por exemplo, o grau de estabilidade das preferências de cada aluno em relação a determinados estilos.

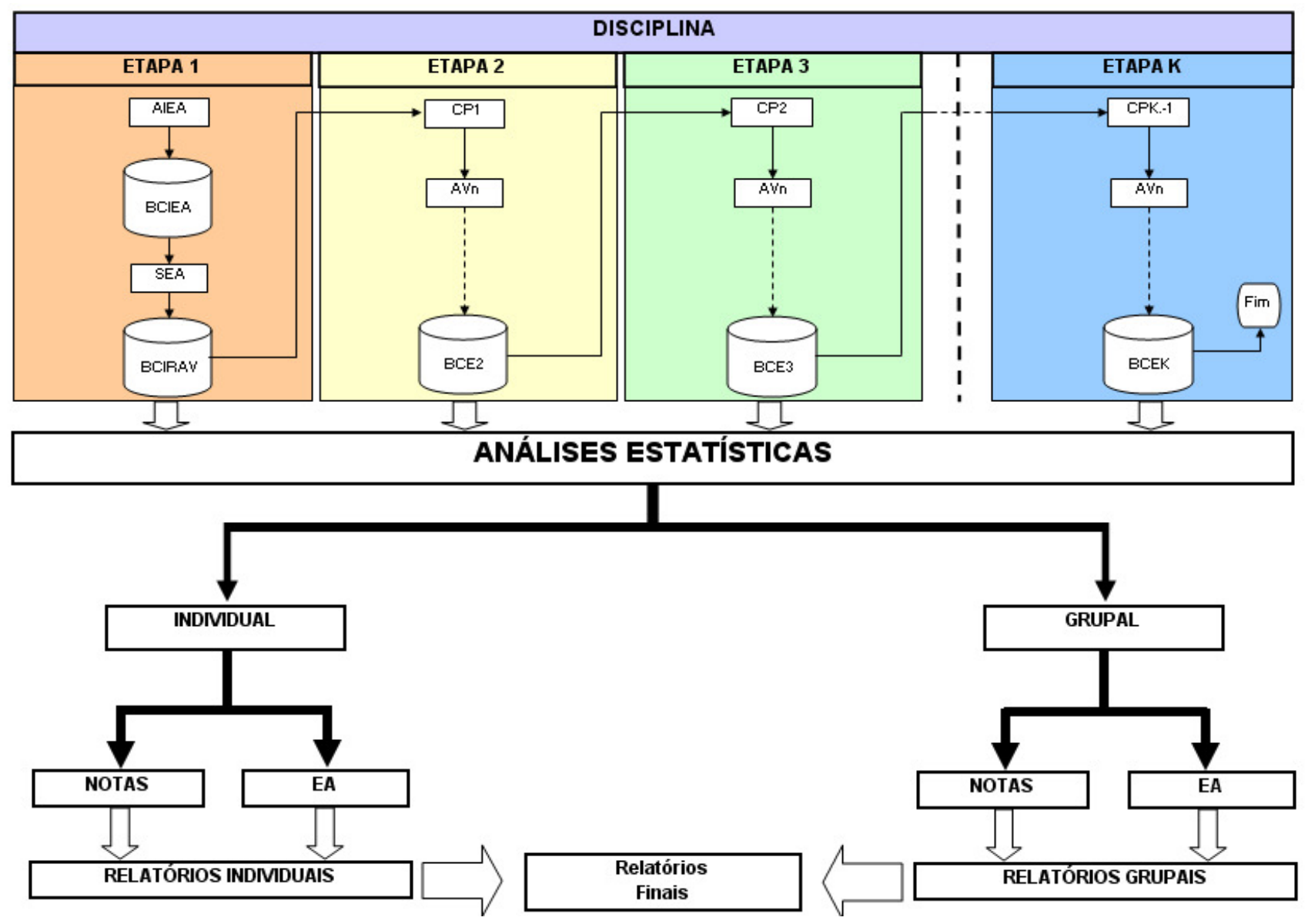

Figura 3: Representação dos relatórios gerados na proposição para uma disciplina. 
Além das análises individuais, outras avaliações estatísticas podem ser obtidas com base no histórico dos grupos formados. Para isso, são utilizados os mesmos indicadores estatísticos definidos para as análises individuais. Esses indicadores podem ser usados tanto para analisar os desempenhos de cada grupo ao longo das avaliações, como para fazer comparações entre os grupos. Outros indicadores, como valores máximo e mínimo, podem ser utilizados para relatar as notas máxima e mínima, contribuindo para avaliar a amplitude de variação das notas de cada grupo. Com os resultados obtidos, é possível efetuar comparações entre os grupos, constatando, por exemplo, aqueles que obtiveram os melhores e piores desempenhos, o que permite identificar os grupos de alunos que tendem a requerer maior atenção por parte do professor.

No que se refere aos indicadores grupais associados com os estilos de aprendizagem, pode-se obter, principalmente, medidas relacionadas com a média de alunos presentes em cada grupo e as variações ocorridas no mesmo.

\section{Conclusões}

No sistema educacional estão inseridos os cursos de educação presencial e a distância, que podem ser realizados com auxílio de ambientes virtuais de ensino e aprendizagem (AVEAs).

Apesar da utilização desses ambientes nas diversas modalidades de ensino estar em ampla expansão no mundo, é pertinente ressaltar que, para aumentar a eficiência do processo ensino-aprendizado, é fundamental adotar procedimentos operacionais adequados aos mesmos.

Diante disso, foi desenvolvida proposição que, a partir de procedimentos operacionais associados com o uso de recursos computacionais, possa ser efetivamente implementada em AVEAs. No entanto, sobre a proposição em questão, é necessário salientar que ela pode ser um tanto complexa por três razões principais: 1) os cursos oferecidos nesses ambientes de ensino, em geral, envolvem amplo número de usuários, que tendem a apresentar características muito heterogêneas, decorrentes de fatores das mais diversas naturezas (e.g., fatores sociais e econômicos); 2) pelo fato de que grande parte desses cursos já possui uma cultura histórica de ensino, existindo, assim, barreiras importantes, tanto em termos de recursos humanos quanto materiais, associadas com a promoção de mudanças no processo educacional; e 3) pela própria existência de complexidades operacionais de implementação da proposta, que estão vinculadas, sobretudo, com os seguintes pontos: definições das formas de ajustes dos conteúdos didáticos e de elaboração das avaliações visando a atender diferentes grupos de alunos, de acordo com distintos estilos de aprendizagem; exigências de modificações estruturais e funcionais importantes nos AVEAs existentes; e necessidade de desenvolvimento de um sistema computacional, que automatize todo o processo da referente proposta.

Apesar das dificuldades operacionais, fica evidente que, para melhorar a eficiência do processo ensino-aprendizado em AVEAs, a definição de estratégias de ensino de acordo com os diferentes estilos de aprendizagem dos alunos pode constituir aspecto fundamental. Nessa perspectiva, a proposta apresentada neste trabalho representa alternativa importante no sentido de contribuir com melhorias para o planejamento e a execução das mais diversas atividades pedagógicas realizadas nesses ambientes.

A respeito da implementação adequada da proposta discutida para um determinado AVEA, resumidamente podese destacar que o mesmo deveria apresentar alguns aspectos fundamentais: 1) estar organizado por disciplinas; 2) possuir um banco de dados para armazenamento e gerenciamento das questões; 3) possuir recursos que permitam a inclusão de cada questão de maneira atrelada ao grupo de estilos de aprendizagem que ela pertence; 4) permitir a inclusão do questionário ILS para a identificação inicial dos estilos de aprendizagem dos alunos; 5) antes que o aluno seja avaliado pelo ILS, restringir o seu acesso à qualquer atividade proposta no ambiente; 6) dispor de um banco de dados (ou arquivo) que armazene os estilos de aprendizagem de cada aluno, identificados durante os processos de avaliação (ILS) e reavaliações (RAVs); e 7) dispor de um meio de armazenamento para as questões e respostas das RAVs. Relacionado com esses aspectos, cabe assinalar que, ao analisar alguns AVEAs (e.g., Moodle e Teleduc), observou-se que a maioria deles está organizada por disciplinas e possui recursos para armazenamento de questões de avaliação da aprendizagem. Porém, os demais aspectos listados, em geral, necessitam ser estruturados.

Finalmente, é pertinente ressaltar que, uma das formas para viabilizar tecnicamente a automação desta proposta está associada com o desenvolvimento de um sistema multiagentes (SMA). Nessa linha, conforme apresentado em Lazzarotto [21], a partir da utilização da linguagem de modelagem MAS-ML, foi desenvolvido um modelo de sociedade de agentes autônomos, que contempla aspectos estruturais e dinâmicos. Com a definição de vários agentes, ficou evidenciada a possibilidade prática de construir um modelo de SMA que, por meio de autonomia e inteligência, contemplasse mecanismos para tornar operacionalizáveis os recursos computacionais propostos, visando a aumentar a eficiência do processo ensino-aprendizado em AVEAs.

\section{Agradecimentos}


Este trabalho foi parcialmente financiado pela FAPEMIG.

\section{Referências}

[1] F. Bica. Eletrotutor III: Uma Abordagem Multiagente para o Ensino à Distância. Dissertação de Mestrado, Universidade Federal do Rio Grande do Sul, Porto Alegre, Janeiro 2000.

[2] B. S. Bloom, J. T. Hastings, G. F. Madeus. Handbook on Formative and Summative Evaluation of Student Learning. McGraw, New York, 1971.

[3] P. Brusilovsky, C. Karagiannidis, D. Sampson. Layered Evaluation of Adaptive Learning Systems; Int. J. Cont. Engineering Education and Lifelong Learning, 14(4/5):402-421, 2004.

[4] P. Brusilovsky, P. Miller. Web-based Testing for Distance Education. In Proceedings of the WebNet World Conference on the $W W W$ and Internet. páginas 149-154, Chesapeake, VA: AACE. 1999.

[5] T. C. S. Cerqueira. Estilos de Aprendizagem em Universitários. Tese de Doutorado, Faculdade de Educação da Universidade Estadual de Campinas, Campinas, Fevereiro 2000.

[6] A. L. Cervo, P. A. Bervian. Metodologia Científica. Makron Books, São Paulo, 1996.

[7] L. Depresbiteris. O Desafio da Avaliação da Aprendizagem: dos Fundamentos a uma Proposta Inovadora. EPU, São Paulo, 1989.

[8] C. C. L. Dias, I. Gasparini, , A. Kemczinski. Identificação dos Estilos Cognitivos de Aprendizagem através da Interação em um Ambiente EAD. In Anais do XVII Workshop sobre Educação em Computação, XXIX Congresso da Sociedade Brasileira de Computação, Bento Gonçalves, 2009 (to appear).

[9] D. D. Diniz. A Interação no Ensino à Distância sob a Ótica dos Estilos de Aprendizagem. Dissertação de Mestrado, Escola de Engenharia de São Carlos da Universidade de São Paulo, São Carlos, Outubro 2007.
[10] J. P. Emiliano. Javal: Modelo de Ambiente de Avaliação Remota Multiagente Baseada em Tutores Embarcados. Dissertação de Mestrado, Universidade Federal do Rio Grande do Sul, Porto Alegre, Março 2002.

[11] R. M. Felder. Author's Preface to Learning and Teaching Styles in Engineering Education. 2002. http://www4.ncsu.edu/unity/lockers/users/f /felder/public/Papers/LS-1988.pdf. Jul. 2008.

[12] R. M. Felder. Reaching the Second Tier Learning and Teaching Styles in College Science Education. Journal College Science Teaching. 23(5):286-290, 1993.

[13] R. M. Felder, R. Brent. Problemas em sala de aula? Ensino Efetivo: Uma Oficina. UFV, Viçosa, 1999.

[14] R. M. Felder, L. Silverman. Learning and Teaching Styles in Engineering Education. Engineering Education. 78(7):674-681, 1988.

[15] R. M. Felder, B. A. Soloman. Index of Learning Styles. 1991. http://www.ncsu.edu/felderpublic/ILSpage.html. Jul. 2008.

[16] J. R. Guedes, C. L. Guedes. Produção de Software Educativo Através de um Projeto Interdisciplinar. In Anais do IV Congresso Brasileiro de Computação, Itajaí, 2004. http://www.dcc.unesc.net/sulcomp/06/artig os/sessaoOral/22007.pdf. Mai 2008.

[17] R. A. N. Granito. Educação a Distância e Estilos de Aprendizagem: Elaboração de um Protocolo de Qualidade para Ambientes Virtuais de Ensino. Dissertação de Mestrado, Faculdade de Economia, Administração e Contabilidade da Universidade de São Paulo, Ribeirão Preto, 2008.

[18] C. Hadji. Avaliação Desmistificada. Artmed, Porto Alegre, 2001.

[19] A. C. Kalatzis. Aprendizagem Baseada em Problemas em uma Plataforma de Ensino a Distância com o Apoio dos Estilos de Aprendizagem: uma Análise do Aproveitamento dos Estudantes de Engenharia. Dissertação de Mestrado, Escola de Engenharia de São Carlos da Universidade de 
São Paulo, São Carlos, Outubro 2008.

[20] G. Lawrence. People Types and Tiger Stripes: a Practical Guide to Learning Styles. Center for Applications of Psychological Type, Gainesville, 1983.

[21] L. L. Lazzarotto. Sistemas Multiagentes na Avaliação Pedagógica e na Detecção do Perfil Cognitivo dos Alunos: Proposta de Modelo para Uso em Ambientes de Ensino a Distância. Dissertação de Mestrado, Universidade Federal de Viçosa, Viçosa, Março 2010.

[22] C. C. Luckesi. Avaliação da Aprendizagem Escolar: Estudos e Proposições. Cortez, São Paulo, 1998.

[23] M. T. Massetto. Mediação Pedagógica e o Uso da Tecnologia. In J. M. Moran, (ed.) Novas Tecnologias e Mediação Pedagógica, páginas 100-173, Papirus, Campinas. 2000.

[24] C. R. R. O. Murad. Descompasso entre Estilos de Ensino/Aprendizagem e os Objetivos dos Alunos. Dissertação de Mestrado, Instituto de Estudos da Linguagem da Universidade Estadual de Campinas, Campinas, 2004.

[25] S. R. M. Oliveira, A. C. Kalatzis, S. J. Bachega, J. S. Kurumoto, E. M. dos Santos, E. W. Cazarini. Proposta Metodológica para Aperfeiçoamento de Planejamento em EAD Utilizando Estilos de Aprendizagem e Competências Requeridas: Aplicação ao Caso da Engenharia de Produção. In: Anais do XIII Congresso Internacional de Educação a Distância, Curitiba, 2007.

[26] P. Perrenoud. Avaliação: da Excelência à Regulação das Aprendizagens - Entre Duas Lógicas. Artes Médicas Sul, Porto Alegre, 1999.

[27] H. V. da Rocha, J. L. Otsuka, C. E. F. Freitas, T. B. Ferreira. Avaliação Online: o Modelo de Suporte Tecnológico do Projeto Teleduc. In M. Silva, E. Santos, (eds.) Avaliação da Aprendizagem em Educação Online, páginas 347-368, Edições Loyola, Ipiranga. 2006.

[28] B. Willis. Distance Education at a Glance. http://www.uidaho.edu/eo/distglan. Mar 2008. 